Research Paper

\title{
Safety and efficacy of drug-eluting bead transarterial chemoembolization with CalliSpheres $₫$ microsphere for hepatocellular carcinoma with portal vein tumor thrombus: a preliminary study
}

Tan-Yang Zhou 1,2, Sheng-Qun Chen 1,3, Hong-Liang Wang1,2, Sheng-Ming Weng', Guan-Hui Zhou',2, Yue-Lin Zhang1,2, Chun-Hui Nie1,2, Tong-Yin Zhu ${ }^{1,2}$, Bao-Quan Wang1,2, Zi-Niu Yu1,2, Li Jing1,2, Feng Chen ${ }^{\circledR}$, Jun-Hui Sun $1,2,3 \bowtie$

1. Hepatobiliary and Pancreatic Interventional Treatment Center, Division of Hepatobiliary and Pancreatic Surgery, The First Affiliated Hospital, Zhejiang University School of Medicine, Hangzhou, Zhejiang Province, China.

2. Zhejiang Provincial Research Center for Diagnosis and Treatment of Hepatobiliary Diseases, Hangzhou, Zhejiang Province, China

3. Zhejiang Clinical Research Center of Hepatobiliary and Pancreatic Diseases, Hangzhou 310003, Zhejiang Province, China.

4. Department of Radiology, Jingning County People's Hospital, Lishui, Zhejiang Province, China

5. Department of Radiology, The First Affiliated Hospital, Zhejiang University School of Medicine, Hangzhou, China.

$\triangle$ Corresponding authors: Feng Chen. Department of Radiology, The First Affiliated Hospital, Zhejiang University School of Medicine, Hangzhou, China. Email: chenfenghz@zju.edu.cn; Jun-Hui Sun. Hepatobiliary and Pancreatic Interventional Treatment Center, Division of Hepatobiliary and Pancreatic Surgery, The First Affiliated Hospital, Zhejiang University School of Medicine; Zhejiang Clinical Research Center of Hepatobiliary and Pancreatic Diseases, Hangzhou 310003, Zhejiang Province, China. Email: 1307005@zju.edu.cn.

(C) The author(s). This is an open access article distributed under the terms of the Creative Commons Attribution License (https://creativecommons.org/licenses/by/4.0/). See http://ivyspring.com/terms for full terms and conditions.

Received: 2020.10.18; Accepted: 2021.05.18; Published: 2021.06.01

\begin{abstract}
Objective: To prospectively evaluate the safety and therapeutic effectiveness of drug-eluting beads transcatheter arterial chemoembolization (DEB-TACE) with CalliSpheres $®$ microsphere (CSM) for the treatment of hepatocellular carcinoma (HCC) with portal vein tumor thrombus (PVTT), and to analyze the prognostic factors.

Method: Between November 2015 and November 2017, consecutive 58 HCC patients with PVTT who received DEB-TACE with CSM treatment were prospectively enrolled in this study. The demographic characteristics, adverse events (AEs) and treatment response were collected. Overall survival (OS) and progression-free survival (PFS) were calculated using the Kaplan-Meier method. Univariate and multivariate Cox regression analyses were performed to determine the independent factors correlated with OS.

Results: The objective response rate (ORR) was $79.3 \%$ in terms of tumors and $44.8 \%$ in thrombi. The median PFS and OS of patients were 5.0 months and 9.0 months respectively. The cumulative survival rate at 3-, 6-, 9-, 12-, 18- and 24-month were $94.8 \%, 72.4 \%, 53.4 \%, 41.4 \%, 22.4 \%$ and $19.0 \%$, respectively. In a stepwise multivariate Cox proportional hazards model, the higher Child-Pugh classification (HR=2.279; $95 \% \mathrm{Cl}, 1.042-4.985, \mathrm{p}=0.039)$ and tumor burden $(\mathrm{p}=0.008)$ were the significant predictors of poorer OS after adjustment for known risk factors. The most common clinical AEs were postembolization syndrome (PES) and the most prevalent laboratory toxicity was transient liver function damage.

Conclusion: DEB-TACE with CSM is safe and well-tolerated in HCC patients with PVTT, and reveals a favorable preliminary clinical outcome. The higher Child-Pugh classification and liver tumor burden are independent prognostic factors associated with poor survival for HCC patients with PVTT treated by DEB-TACE with CSM.
\end{abstract}

Key words: Hepatocellular carcinoma, Chemoembolization, Therapeutic, Microspheres, Prognosis 


\section{Introduction}

Hepatocellular carcinoma (HCC) is the most common tumor in the hepatobiliary system and is the third leading cause of cancer death all around the world [1]. HCC tends to invade the intrahepatic vasculature, especially the portal vein and form a portal vein tumor thrombus (PVTT)[2]. PVTT is associated with a dismal prognosis for HCC patients, with a median survival time of only 2.7 months without any interventions [3].

Current therapeutic approaches for HCC patients with PVTT include hepatectomy, transcatheter arterial chemoembolization (TACE), radiotherapy, systematic chemotherapy according to the national guidelines in China [4]. Among these available treatment approaches, TACE is one of the most commonly used techniques to manage unresectable HCC with PVTT [5]. However, most of the previous studies mainly using conventional TACE (cTACE), which the rationale behind is that the intra-arterial chemotherapy using lipiodol and chemotherapeutic agents, followed by selective vascular embolization, will result in a strong cytotoxic effect combined with ischemic necrosis. Recently, drug-eluting beads transcatheter arterial chemoembolization (DEB-TACE) was developed to treat advanced HCC and was known to deliver higher doses of chemotherapeutic agents and to prolong contact time with the tumor [6]. However, there are limited data are available concerning the use of DEB-TACE in HCC patients with PVTT.

CalliSpheres ${ }^{\circledR}$ microsphere (CSM), is a newly invented drug-eluting beads (DEB) in China, which is structured on a polyvinyl alcohol hydrogel modified with sulfonate groups. Many pieces of research have proved that it can load more kinds of chemotherapy drugs and has a higher drug-loading efficiency compared with other DEBs [7-9]. According to several recent studies, unresectable HCC patients who receive DEB-TACE treatment using CSM have better treatment response, prolonged overall survival (OS) and reduced adverse events (AEs) compared with CTACE[10-11]. Therefore, the purpose of this study was to investigate the treatment efficacy and safety of DEB-TACE using CSM and to identify important prognostic factors related to the survival of HCC patients with PVTT.

\section{Methods}

\section{Patients}

Between November 2015 and November 2017, consecutive 58 HCC patients with PVTT who received DEB-TACE treatment using CSM in the First Affiliated Hospital, Zhejiang University School of
Medicine were prospectively enrolled in this study. The inclusion criteria were as follows: (1) diagnosed as HCC confirmed either by clinical and histopathological examinations according to the American Association for the Study of Liver Diseases (AASLD) [12]; (2) PVTT was confirmed by enhanced Computed Tomography (CT) or enhanced Magnetic Resonance Imaging (MRI) according to Chinese Expert Consensus on Multidisciplinary Diagnosis and Treatment of Hepatocellular Carcinoma with Portal Vein Tumor Thrombus (2018 Edition) [5] and the imaging features of PVTT included solid lesions within the portal vein in all the phases of intravenous enhanced 3-phase computed tomography, especially with an enhancement of contrast in the arterial phase and washout in the portal venous phase of the procedure. PVTT was divided according to Cheng's PVTT classification system into the following categories [5]: type I, tumor thrombus involve the segmental branches of the portal vein or above; type II, the right/left portal vein; type III, the main portal vein; and type IV, the superior mesenteric vein; (3) with Eastern Cooperative Oncology Group (ECOG) performance status $\leq 1$ and Child-Pugh classification A or B; (4) medical records were completely reserved and available. The exclusion criteria included: (1) patients with obvious hepatic arteriovenous fistula showed in CT/MRI or decompensated cirrhosis; (2) patients who accompanied with severe cardiovascular diseases or other malignancies; (3) patients whose medical records were incomplete; (4) patients with a history of either locoregional therapies or systemic treatments before enrolled. The present study was approved by the Institutional Review Board of the First Affiliated Hospital, Zhejiang University School of Medicine, with informed consent obtained from all patients.

\section{Data collection}

All data were extracted from the electronic medical records. Baseline demographics and clinical characteristics included age, gender, ECOG performance status, Child-Pugh classification, viral etiology, Hepatitis B Virus (HBV)-DNA, liver cirrhosis, ascites, number of HCC nodules, macroscopic classification, maximum tumor diameter, tumor burden, serum alpha-fetoprotein (AFP), PVTT classification and extrahepatic metastasis. Besides, treatment response, routine laboratory tests (including complete blood count, biochemical parameters) before and after treatment (4-6 weeks) were also collected.

The tumor burden, the percentage of total tumor in the liver, was independently determined by an abdominal radiologist with at least 3 years of 
experience in liver imaging on a workstation (Advantage Windows ${ }^{\circledR}$,VolumeShare 4, GE Healthcare, Milwaukee, Wisc., USA) using dedicated software (Volume Viewer ${ }^{\circledR}$, GE Healthcare, Milwaukee, Wisc., USA).

\section{DEB-TACE procedures}

Before the initiation of DEB-TACE, the concentrated solution of epirubicin was prepared with $4 \mathrm{ml}$ of sterile water for injection and $80 \mathrm{mg}$ of epirubicin hydrochloride, and the concentration was $20 \mathrm{mg} / \mathrm{ml}$. Then the CSM (Jiangsu Hengrui Medicine Co., Ltd., Jiangsu Province, China) with sizes of 100$300 \mu \mathrm{m}$ were loaded with epirubicin as follows: firstly, the CSM and sterile water were extracted by a $20 \mathrm{~mL}$ syringe and inverted placed for 5 min until the CSM were totally precipitated, then pushed out the supernatant liquor. Subsequently, the concentrated solution of epirubicin was mixed with the CSM using a tee joint and then stored by a syringe, and the syringe containing the mixture of CSM and epirubicin solution was placed at room temperature and shaken gently every 5 min until almost all epirubicin were loaded (loading time more than $30 \mathrm{~min}$ ). After that, the nonionic contrast medium (iodixanol [320 mg $\mathrm{I} / \mathrm{mL}]$, Jiangsu Hengrui Medicine, Jiangsu, China) was added into the mixture as a 1:1 ratio and the mixture were kept still for $5 \mathrm{~min}$ for further application.

It was recommended to use $2.4-\mathrm{F}$ to $2.8-\mathrm{F}$ microcatheters for the transcatheter embolization of tumor feeders, to perform cone-beam CT as soon as deemed necessary, and to inject the beads slowly (ideally $1 \mathrm{~mL} / \mathrm{min}$ ) through a $1-\mathrm{mL}$ syringe until the reduced flow of the feeding artery with the conventional method of two to five heartbeats to clear the contrast column from the microcatheter tip. Additional embolic material such as polyvinyl alcohol (PVA) particles or tris-acryl gelatin microspheres (Embosphere ${ }^{\circledR}$ Microspheres, BioSphere Medical, Rockland, MA) was used if the beads were completely delivered before the endpoint of embolization was reached. Superselective embolization as much as possible was strongly recommended to avoid injury of nontumoral liver parenchyma.

All patients received routine tests including complete blood count, biochemical indexes, coagulation function, tumor markers and liver enhanced CT or MRI before and after DEB-TACE. Besides, the necessary symptomatic and supportive treatments such as actively liver-protecting treatment, pain relief, antiemetics and prophylactic anti-infection were provided to all patients after DEB-TACE until they fully recovered and discharged.

\section{Safety assessment}

The safety assessment was evaluated based on a procedure related to AEs grading. AEs were assessed within 1 month after the procedure and included clinical and laboratory toxicity. The grading of AEs were defined as low (grades 1-2) or high (grades 3-4) according to Common Terminology Criteria for Adverse Events v5.0 (CTCAE v5.0).

\section{Response assessment and follow up}

Liver enhanced CT or MRI examination was performed at 4-6 weeks after DEB-TACE, and the treatment response was assessed according to modified Response Evaluation Criteria in Solid Tumors (mRECIST), which included complete response $(\mathrm{CR})$, partial response (PR), stable disease (SD) and progressive disease (PD). The PVTT radiologic response was assessed as a complete response $(\mathrm{CR}$, without any enhancement or complete disappearance), partial response ( $\mathrm{PR},>50 \%$ decrease in the enhancement region or the thrombus diameter), stable disease (SD, $<50 \%$ decrease or $<25 \%$ increase in the enhancement region or the thrombus diameter or cavernous transformation), and progressive disease (PD, $>25 \%$ increase in the thrombus diameter or newly developed PVTT), based on the criteria described by Yoon et al [13]. Overall response rate (ORR) was defined as the rate of CR+PR. Moreover, patients were followed up by calls, outpatient service and hospitalizations for 6-30 months after DEB-TACE treatment, and the last follow-up date was Jun 30th, 2020. PFS was defined as the time elapsed between DEB-TACE treatment initiation and tumor progression or death from any cause, with censoring of patients who received other therapies or were lost to follow-up. OS was defined as the period from the first DEB-TACE treatment to the date of death from any cause or until the date of the last follow-up.

\section{Statistical analysis}

Count data were expressed as count (percentage), and the measurement data were presented as mean \pm standard deviation. Comparison of measurement data between pretreatment and posttreatment was determined by paired t-test. OS was analyzed using the Kaplan-Meier method and compared using the log-rank test. The multivariate Cox proportional hazard modeling was performed to identify independent prognostic factors based on the adjusted hazard ratio and its associated 95\% confidence interval (CI). All statistical analyses were performed using SPSS 22.0 (IBM,Chicago, IL, USA). For all tests, $\mathrm{P}<0.05$ was considered statistically significant. 


\section{Results}

\section{Patients' baseline characteristics}

Among the 58 patients, 53 were men (91.4\%) and 23 patients aged $\geq 60$ years $(39.7 \%)$. The majority of patients were ECOG $1(62.1 \%)$ in performance status and with Child-Pugh classification A liver function (81\%). Approximate two-thirds of patients had multiple tumor nodules and type I/II PVTT. There were only $31 \%$ of patients with low tumor burden $(\leq 30 \%)$ and 47 patients without extrahepatic metastasis. In 11 patients with extrahepatic metastasis, ten suffered from pulmonary metastasis and one had abdominal lymph node metastasis. The baseline characteristics of patients are presented in Table 1.

Table 1. Patients' baseline characteristics $(\mathrm{N}=58)$.

\begin{tabular}{|c|c|c|c|c|c|}
\hline Characteristics & No. & Percentage & Characteristics & No. & Percentage \\
\hline Age (years) & & & 2 & 2 & 3.4 \\
\hline$\geq 60$ & 23 & 39.7 & $\geq 3$ & 39 & 67.3 \\
\hline$<60$ & 35 & 60.3 & Tumor type & & \\
\hline Gender & & & Nodular & 11 & 19 \\
\hline Male & 53 & 91.4 & Massive & 11 & 19 \\
\hline Female & 5 & 8.6 & Diffuse & 36 & 62 \\
\hline $\begin{array}{l}\text { ECOG performance } \\
\text { status }\end{array}$ & & & $\begin{array}{l}\text { Maximum tumor size } \\
(\mathrm{cm})\end{array}$ & & \\
\hline 0 & 22 & 37.9 & $<5$ & 7 & 12.1 \\
\hline 1 & 36 & 62.1 & $\geq 5,<10$ & 28 & 48.3 \\
\hline $\begin{array}{l}\text { Child-Pugh } \\
\text { classification }\end{array}$ & & & $\geq 10$ & 23 & 39.6 \\
\hline A & 47 & 81 & Tumor burden & & \\
\hline B & 11 & 19 & $\leq 30 \%$ & 18 & 31 \\
\hline Viral etiology & & & $\leq 50 \%$ & 20 & 34.5 \\
\hline $\mathrm{HBsAg}(+)$ & 52 & 90 & $>50 \%$ & 20 & 34.5 \\
\hline $\operatorname{HBsAg}(-)$ & 6 & 10 & Serum AFP (ng/ml) & & \\
\hline HBV-DNA(IU/ml) & & & $<200$ & 22 & 37.9 \\
\hline $\begin{array}{l}\text { Below the detection } \\
\text { limit }\end{array}$ & 26 & 44.8 & $\geq 200,<400$ & 3 & 5.2 \\
\hline$<10^{4}$ & 13 & 22.4 & $\geq 400$ & 33 & 56.9 \\
\hline$\geq 10^{4}$ & 19 & 32.8 & Type of PVTT & & \\
\hline Liver cirrhosis & & & I & 16 & 27.6 \\
\hline Yes & 56 & 96.6 & II & 21 & 36.2 \\
\hline No & 2 & 3.4 & III & 16 & 27.6 \\
\hline Ascites & & & IV & 5 & 8.6 \\
\hline Yes & 11 & 19 & $\begin{array}{l}\text { Extrahepatic } \\
\text { metastasis }\end{array}$ & & \\
\hline No & 47 & 81 & Yes & 11 & 19 \\
\hline No. of HCC nodules & & & No & 47 & 81 \\
\hline 1 & 17 & 29.3 & & & \\
\hline
\end{tabular}

\section{Treatments}

The 58 patients included in the study received 71 cycles of DEB-TACE. The median number of TACE sessions per patient was 1.2 (range, 1-3).The vast majority of the patients $(82.8 \%)$ received one cycle and only 3 patients receive 3 cycles. In addition, of all the 58 patients, 34 cases only underwent DEB-TACE treatment, 11 cases received DEB-TACE combined with sorafenib, 4 cases underwent DEB-TACE combined with surgery, 3 cases received DEB-TACE combined with liver transplantation, 2 cases received DEB-TACE combined with portal vein iodine 125 particle stent, 2 cases received DEB-TACE combined with sorafenib and surgery, 1 case underwent DEB-TACE combined with ${ }^{131}$ I-metuximab injection, and 1 case underwent DEB-TACE combined with intraperitoneal hyperthermic perfusion chemotherapy.

\section{Treatment response evaluation}

Treatment response of patients are evaluated according to the mRECIST, which showed that 3 (5.2\%) and 43 (74.1\%) patients achieved CR and PR respectively, with an ORR $79.3 \%$ regarding tumors; 2 (3.4\%) and 24 (41.4\%) patients achieved CR and PR respectively, with an ORR $44.8 \%$ in terms of tumor thrombus (Table 2).

Table 2. Response to treatment.

\begin{tabular}{|c|c|c|c|c|c|}
\hline Items & CR & PR & SD & PD & ORR \\
\hline Tumor & $3(5.2)$ & $43(74.1)$ & $9(15.6)$ & $3(5.1)$ & $46(79.3)$ \\
\hline Tumor Thrombus & $2(3.4)$ & $24(41.4)$ & $25(43.1)$ & $7(12.1)$ & $26(44.8)$ \\
\hline
\end{tabular}

\section{Survival evaluation of patients}

The Kaplan-Meier curve was drawn to analyze the PFS and OS of patients, which revealed that the median PFS and OS of patients were 5.0 months and 9.0 months. The 3-, 6-, 9-, 12-, 18- and 24-month OS rates were $94.8 \%, 72.4 \%, 53.4 \%, 41.4 \%, 22.4 \%$ and $19.0 \%$, respectively (Figure 1). Meanwhile, higher Child-Pugh classification (Figure 2A) and tumor burden (Figure 2B) were observed to be associated with worse OS.

\section{Cox's proportional hazards regression model analysis of factors affecting OS}

To analyze factors affecting OS, univariate Cox's proportional hazards regression model was applied, which revealed that Child-Pugh classification $(\mathrm{P}=0.004)$, extrahepatic metastasis $(\mathrm{P}=0.022)$ and larger maximum tumor size $(\mathrm{P}=0.003)$, as well as tumor burden $(\mathrm{P}=0.001)$ were associated with poorer OS (Table 3). To further explore independent factors for predicting OS, multivariate Cox's proportional hazards regression model was utilized, and it showed that higher Child-Pugh classification $(\mathrm{P}=0.032)$ and tumor burden $(\mathrm{P}=0.002)$ were independent factors for predicting worse OS (Table 3). 

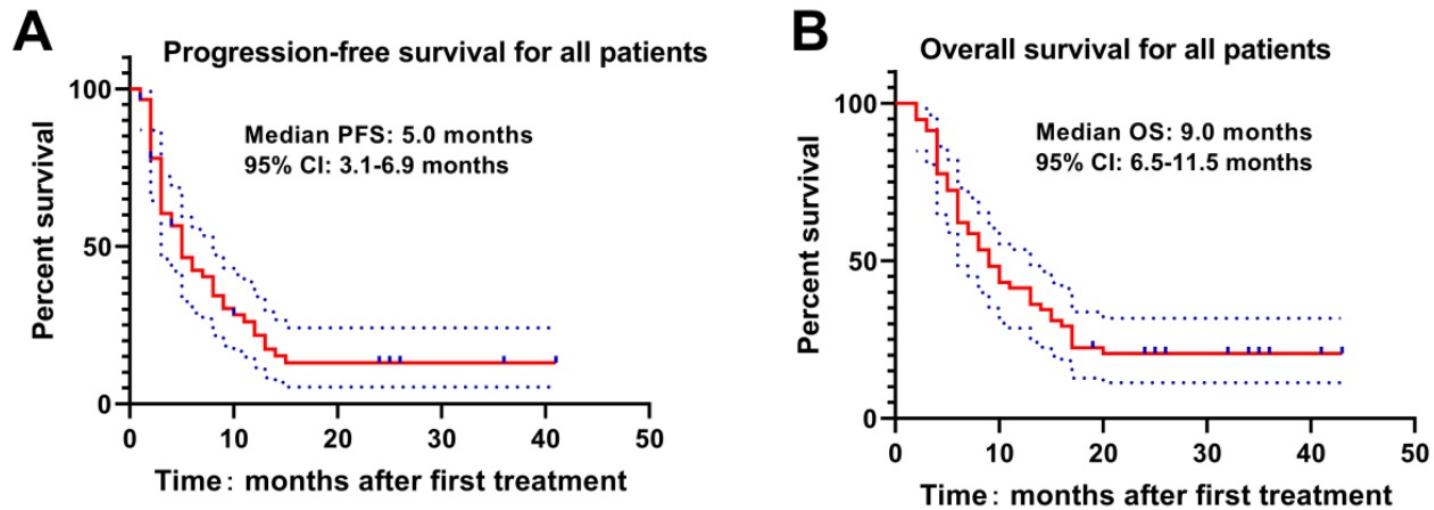

Figure 1. PFS and OS of HCC patients with PVTT underwent DEB-TACE with CSM. The median PFS(A) and OS(B) were 5.0 months and 9.0 months respectively in HCC patients with PVTT underwent DEB-TACE with CSM.

\section{Comparison of OS in subgroup analysis}
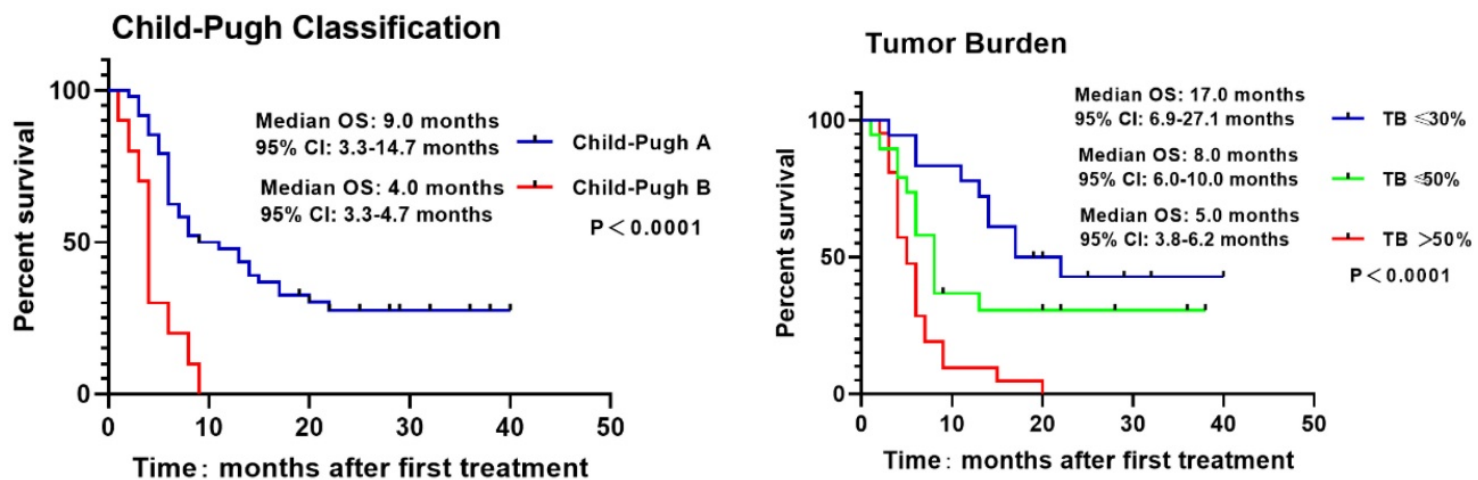

Figure 2. Associations of Child-Pugh classification and tumor burden with OS. The higher Child-Pugh classification(A) and the higher tumor burden (B) were associated with poorer OS. Survival profiles were illuminated with Kaplan-Meier method. OS, overall survival; TB, Tumor Burden

\section{Assessment of safety}

The safety assessment was performed one day after the procedure for laboratory toxicity and within one week for clinical toxicity. The detailed safety assessment is shown in Table 4. The most common AEs were moderate fever (91.4\%), abdominal pain $(75.9 \%)$ and nausea $(70.7 \%)$, which were known as the postembolization syndrome (PES). Almost all patients had suffered transient liver function damage after the procedure, with mild to moderately elevated alanine transaminase $(50.0 \%)$ and bilirubinemia $(63.8 \%)$, but high-grade aspartate transaminase elevation (58.6\%). No deaths occurred within 1 month after treatment. However, three patients died due to hepatic failure $(n=1)$ or gastrointestinal bleeding $(n=2)$ within 3 months after the procedure.

\section{Discussion}

There is a high incidence of PVTT in HCC patients, which is associated with a poor prognosis [14-15]. But at present, treatment strategies for HCC patients with PVTT remain controversial. According to the Barcelona Clinic for Liver Cancer (BCLC) staging system, PVTT is recognized as an advanced stage disease (BCLC-C) and systemic therapy such as sorafenib is the only recommended therapeutic strategy by the AASLD guidelines [12], but in the Asia-Pacific region TACE is an essential method for HCC patient with PVTT in many medical centers [16-17]. In a recent study, the median survival time of TACE (7.49 months) was similar to the result of the SHARP trial (8.1 months) in patients with HCC and vascular invasion [17-18], which were slightly lower than that of our study. Moreover, in the SHARP trial, $95 \%$ of patients were categorized as Child-Pugh classification A liver function and tumor thrombosis was limited as macroscopic vascular invasion. Hepatic resection (HR) is a safe and effective treatment for HCC with PVTT when patients are carefully selected [19]. HR can eradicate both the main tumor and satellite tumors as well as PVTT to reduce the pressure on the portal vein, preventing the occurrence of refractory ascites and bleeding of esophageal varices, protecting liver function, and reducing tumor burden as well as intrahepatic and extrahepatic metastasis of HCC [20]. But type III or IV PVTT may lead to intraoperative difficulties in resecting the thrombi. Meanwhile, portal vein wall invasion may lead to thrombi residue and a high risk 
of postoperative recurrence. A systematic review about the safety and efficacy of HR for treating HCC involving a single large tumor $(>5 \mathrm{~cm})$ or multiple tumors, or for treating HCC involving macrovascular invasion shows the median OS investigating HCC with macrovascular invasion was approximately $50 \%$ at 1 year and $18 \%$ at 5 years [19], which were significantly higher than those in our study. And those results may benefit from that there were no extrahepatic metastasis for patients suit for HR. However, in our study, there were $19.0 \%$ of the patients accompanied with extrahepatic metastasis and $36.2 \%$ of the patients had tumor thrombosis extending to the main portal vein, the splenic vein, or the superior mesenteric vein (grades III/IV PVTT).

Table 3. Univariate and stepwise multivariate Cox's proportional hazards regression model analysis factors affecting OS.

\begin{tabular}{|c|c|c|c|c|c|c|}
\hline \multirow[t]{2}{*}{ Variable } & \multicolumn{3}{|c|}{ Univariate analysis } & \multicolumn{3}{|c|}{ Multivariate analysis } \\
\hline & $\overline{\text { HR }}$ & $95 \% \mathrm{CI}$ & $P$ & HR & $95 \% \mathrm{CI}$ & $P$ \\
\hline Age & 0.970 & $0.485-1.940$ & 0.932 & - & - & - \\
\hline Gender (male/female) & 1.387 & $0.331-5.807$ & 0.654 & - & - & - \\
\hline $\begin{array}{l}\text { Etiology(hepatitis } \\
\text { infection/other) }\end{array}$ & 0.760 & $0.267-2.167$ & 0.608 & - & - & - \\
\hline HBV-DNA detectable(yes/no) & 1.170 & $0.805-1.700$ & 0.412 & - & - & - \\
\hline $\begin{array}{l}\text { Child-Pugh classification } \\
\text { (B/A) }\end{array}$ & 2.986 & $1.412-6.316$ & 0.004 & 2.279 & $1.042-4.985$ & 0.039 \\
\hline $\operatorname{ECOG}(0 / 1)$ & 0.713 & $0.347-1.464$ & 0.357 & - & - & - \\
\hline $\begin{array}{l}\text { Extrahepatic metastasis } \\
\text { (yes/no) }\end{array}$ & 2.481 & $1.142-5.393$ & 0.022 & - & - & - \\
\hline Maximum tumor size & & & 0.009 & & & \\
\hline$<5$ & Ref & & & & & \\
\hline$\geq 5,<10$ & 1.986 & $0.447-8.819$ & 0.367 & & & \\
\hline$\geq 10$ & 5.081 & $1.173-22.002$ & 0.030 & & & \\
\hline No. of HCC nodules $(\geq 3 /<3)$ & 1.217 & $0.818-1.811$ & 0.333 & - & - & - \\
\hline $\operatorname{AFP}(\geq 400 /<400)$ & 1.168 & $0.810-1.683$ & 0.407 & - & - & - \\
\hline Type of PVTT & & & 0.813 & - & - & - \\
\hline I & Ref & & & & & \\
\hline II & 1.148 & $0.469-2.813$ & 0.762 & & & \\
\hline III & 1.539 & $0.615-3.850$ & 0.357 & & & \\
\hline IV & 1.135 & $0.3000-4.285$ & 0.852 & & & \\
\hline Tumor type & & & 0.141 & - & - & - \\
\hline Nodular & Ref & & & & & \\
\hline Massive & 2.065 & $0.729-5.851$ & 0.172 & & & \\
\hline infiltrative & 0.929 & $0.370-2.328$ & 0.874 & & & \\
\hline Tumor Burden & & & 0.002 & & & 0.008 \\
\hline$\leq 30 \%$ & Ref & & & Ref & & \\
\hline$\leq 50 \%$ & 3.273 & $1.147-9.337$ & 0.027 & 3.300 & $1.152-9.454$ & 0.026 \\
\hline$>50 \%$ & 6.248 & $2.228-17.523$ & 0.000 & 5.380 & $1.873-15.458$ & 0.002 \\
\hline Ascites (yes/no) & 1.898 & $0.850-4.235$ & 0.118 & - & - & - \\
\hline $\begin{array}{l}\text { Data were presented as P value } \\
\text { interval). Factors affecting OS ( } \\
\text { multivariate Cox's proportiona } \\
\text { considered significant. "-" indi } \\
\text { ECOG, Eastern Cooperative Or } \\
\text { HBV; Hepatitis B Virus; HCC, } \\
\text { PVTT, Portal Vein Tumor Thro }\end{array}$ & $\begin{array}{l}\text { HR (h } \\
\text { verall } \\
\text { hazarc } \\
\text { ited th } \\
\text { ology } \\
\text { patoc } \\
\text { ibus. }\end{array}$ & $\begin{array}{l}\text { hazards ratio) } \\
\text { survival) wer } \\
\text { ds regression } \\
\text { hat the factor } \\
\text { Group; HBs } \\
\text { cellular carcin }\end{array}$ & $\begin{array}{l}\text { and } 95 \\
\text { ce dete } \\
\text { analys } \\
\text { was no } \\
\mathrm{Ag}, \mathrm{He} \\
\text { oma; }\end{array}$ & $\begin{array}{l}5 \% \text { CI } \\
\text { rminec } \\
\text { ses. } P \text { V } \\
\text { statis } \\
\text { patitis } \\
\text { AFP, al }\end{array}$ & $\begin{array}{l}\text { (confidence } \\
d \text { by univariat } \\
\text { value }<0.05 \text { wo } \\
\text { tical significar } \\
\text { B surface ant } \\
\text { lpha-fetoprote }\end{array}$ & $\begin{array}{l}\text { te and } \\
\text { as } \\
\text { nce. } \\
\text { tigen } \\
\text { ein; }\end{array}$ \\
\hline
\end{tabular}

Drug-eluting bead TACE (DEB-TACE), a novel TACE that uses microspheres as both drug carriers and embolization agents, possesses several advantages over cTACE, including more constant drug release and better embolization effect [21-23]. Accumulating evidence reveals that DEB-TACE is superior to CTACE in improving treatment response and OS but fewer adverse events of unresectable HCC patients [21, 23-25]. As to its efficacy in treating HCC patients with PVTT, only two studies have been reported [26-27]. In a recent study, $4 \%$ of HCC patients with PVTT achieve CR after DEB-TACE treatment, and the ORR is $26 \%$; besides, median survival is 10 months [26]. In another study, the survival of HCC patients with PVTT is also evaluated after DEB-TACE treatment, which reveals that the median OS is 3.33 months [27]. Nevertheless, only two small-sample studies are unable to fully illustrate the efficacy of DEB-TACE in treating HCC patients with PVTT. Therefore, additional investigations are needed to reveal the clinical response and survival of DEB-TACE in treating HCC patients with PVTT.

Table 4. The main AEs of DEB-TACE with CSM for HCC with PVTT.

\begin{tabular}{lll}
\hline Variables & Grade 1-2 (n, \%) & Grade 3-4 (n, \%) \\
\hline Abdominal pain & $44(75.9)$ & $1(1.7)$ \\
Fever & $53(91.4)$ & $2(3.4)$ \\
Nausea & $41(70.7)$ & 0 \\
Diarrhea & $5(8.6)$ & 0 \\
WBC decreased & $6(10.3)$ & 0 \\
Neutrophils decreased & $2(3.4)$ & 0 \\
HGB decreased & $3(5.2)$ & 0 \\
PLT decreased & $15(25.9)$ & $6(10.3)$ \\
Elevated ALT & $29(50.0)$ & $21(36.2)$ \\
Elevated AST & $22(37.9)$ & $34(58.6)$ \\
Hyperbilirubinemia & $37(63.8)$ & $2(3.4)$ \\
\hline Note: WBC, white blood cell; HBG, hemoglobin; PLT, platelet; ALT, alanine \\
aminotransferase; AST, aspartate aminotransferase; GI, gastrointestinal.
\end{tabular}

CSM, the first DEB-TACE product in China, not only has general advantages of DEB-TACE, but also has higher drug loading efficiency compared with other DEB-TACE products [28]. Since CSM is approved in 2015, it has been widely used in China to treat unresectable HCC patients due to its good characteristics $[7,28]$. Considering that the efficacy of DEB-TACE in treating HCC patients with PVTT remains to be illustrated, especially for DEB-TACE using CSM, we conducted the current study, and found that $5.2 \%$ of tumor achieved CR, and the ORR was $79.3 \%$ for tumor after DEB-TACE treatment; besides, the median PFS and OS of patients were 5.0 months and 9.0 months. The 3-, 6-, 9-, 12-, 18- and 24-month OS rates were $94.8 \%, 72.4 \%, 53.4 \%, 41.4 \%$, $22.4 \%$ and $19.0 \%$, respectively. These findings were similar to our previous study with portal vein stenting combined with iodine- 125 seed strand endovascular implantation followed by TACE for treating HCC patients with PVTT [29]. The efficacy of DEB-TACE in our study was better than that of the two previous studies, which might be explained by that: (1) the better properties of CSM over other DEB-TACE products contributed to the better efficacy; (2) our study only recruited patients with Child-Pugh 
classification A or B liver function, while previous studies also recruit Child-Pugh classification C patients, implying that HCC patients with PVTT in our study had better baseline liver function compared to the previous studies, which might also lead to the better clinical outcomes; (3) there were only 34 cases underwent DEB-TACE monotherapy, and the remaining 24 patients accepted combination therapy including sorafenib, surgery and even liver transplantation, which may prolong OS in our cohort Taken together, our study revealed that DEB-TACE using CSM presented with a favorable clinical efficacy in HCC patients with PVTT.

A lot of risk factors for OS of HCC patients with PVTT have been discovered [29]. A prospective study investigates the prognostic factors of HCC patients with PVTT who receive TACE or conservative treatment, which shows that the presence of main portal vein obstruction, tumor number $\geq 2$, TBIL $\geq 20$ $\mu \mathrm{mol} / \mathrm{L}$ and tumor diameter $\geq 11.1 \mathrm{~cm}$ are associated with worse OS [16]. Another retrospective study including HCC patients with PVTT treated with DEB-TACE or radioembolization (90Y) revealed increased age, the extent of liver involvement and weight to be independent risk factors for poorer OS [26]. More interestingly, a retrospective study discloses that Child-Pugh classification $\mathrm{C}$ and tumor burden $>50 \%$ are independent predictors for poorer OS in HCC patients with PVTT who receive DEB-TACE or CTACE treatment [27]. Consistent with previous studies, our study found that the higher Child-Pugh classification, extrahepatic metastasis and the greater tumor size as well as the higher tumor burden were associated with worse OS; what's more, the higher Child-Pugh classification and tumor burden were independent predictors for poorer OS. The possible reasons might be as follows: (i) Compared to patients at Child-Pugh classification A, patients at stage $\mathrm{B}$ have more dysregulated liver functions and deteriorated quality of life, thus they tend to have poorer OS; (ii) increased tumor burden indicates larger tumor size while less hepatic functional reserve, as a result, patients with increased tumor burden are also more likely to have a poorer OS. Above all, both worse liver function of ChildPugh classification and higher tumor burden were independent risk factors for worse OS.

As for AEs of DEB-TACE in treating HCC patients with PVTT, two studies have been reported [26-27]. In one of the studies, the AEs included pain, nausea and vomiting, which occurred in $10.8 \%$ of patients, and $26 \%$ of patients were dead three months post treatment [26]. In the other study, post-embolization syndrome, encephalopathy, diarrhea and abnormal liver functions were the main
AEs, and $6.3 \%$ of patients were dead one month post treatment [27]. In our study, the most common clinical AEs were PES and the most prevalent laboratory toxicity was transient liver function damage. There were no deaths occurred within 1 month after treatment but three patients died due to hepatic failure or gastrointestinal bleeding within 3 months after the procedure. There could be many reasons for the lower mortality rate compared with previous studies but the main two reasons were the patients in our cohort with Child-Pugh classification A or B liver function and successful superselective catheterization of not only tumor vessels but also the nutrient arteries contributing to tumor thrombosis. Our results indicated that DEB-TACE with CSM had good safety for HCC with PVTT in patients with Child-Pugh classification A or B liver function.

There were some limitations in the current study. Firstly, the study was a single-arm study without comparison, and the sample size was relatively small, thus the randomized, control study with a larger sample size is needed. Second, all of the patients recruited in this study were treatment-naïve patients, so the efficacy and safety of DEB-TACE using CSM in patients with treatment-experienced remained unclear. Third, only a few patients in our study combined with sorafenib, and the most common reason is the high cost that patients cannot afford because sorafenib is not on the list of drugs covered by medical insurance of China until October 2017. Therefore, the treatment outcome of DEB-TACE using CSM combined with sorafenib in the real-world needs further study. At last, we only evaluated the efficacy and safety of DEB-TACE using CSM in Chinese patients, which could not represent other race patients, thus the efficacy and safety of DEB-TACE using CSM in other race patients need further investigated in the future.

In summary, DEB-TACE is safe and well-tolerated in HCC patients with PVTT, and reveals a good preliminary clinical outcome according to our study, although requiring further stringent evaluation. The higher Child-Pugh classification and liver tumor burden are independent prognostic factors associated with poor survival for HCC patients with PVTT treated by DEB-TACE with CMS.

\section{Abbreviations}

DEB: Drug-eluting beads; TACE: Transcatheter arterial chemoembolization; CSM: CalliSpheres ${ }^{\circledR}$ microsphere; HCC: Hepatocellular carcinoma; PVTT: Portal vein tumor thrombus; AASLD: the American Association for the Study of Liver Diseases; mRECIST: modified Response Evaluation Criteria in Solid Tumors; AEs: Adverse events; OS: Overall survival; 
CR: Complete response; ECOG: Eastern Cooperative Oncology Group; ORR: Overall response rate; PFS: Progression free survival; PR: Partial response; SD: Stable disease; PD: Progressive disease; CT: Computed Tomography; MRI: Magnetic Resonance Imaging; PVA: Polyvinyl alcohol.

\section{Acknowledgements}

This work was sponsored by Zhejiang Provincial Natural Science Foundation of China (Grant No.LZ18H180001), National Natural Science Foundation of China (Grant No. 81971713), National S\&T Major Project of China (NO.2018ZX10301201), Grant from Health Commission of Zhejiang Province (JBZX-202004), Research Unit of Collaborative Diagnosis and Treatment For Hepatobiliary and Pancreatic Cancer, Chinese Academy of Medical Sciences (2019RU019) and Research Fund for Interventional Oncology of China Health Promotion Foundation (No.XM_2018_011_0006_01).

\section{Competing Interests}

The authors have declared that no competing interest exists.

\section{References}

1. Torre LA, Bray F, Siegel RL, et al. Global cancer statistics, 2012. CA Cancer J Clin. 2015; 65(2): 87-108.

2. Wei X, Jiang Y, Zhang X, et al. Neoadjuvant Three-Dimensional Conformal Radiotherapy for Resectable Hepatocellular Carcinoma With Portal Vein Tumor Thrombus: A Randomized, Open-Label, Multicenter Controlled Study. J Clin Oncol. 2019; 37(24): 2141-51.

3. Mahringer-Kunz A, Steinle V, Duber C, et al. Extent of portal vein tumour thrombosis in patients with hepatocellular carcinoma: The more, the worse? Liver Int. 2019; 39(2): 324-31.

4. Zhou J, Sun HC, Wang Z, et al. Guidelines for Diagnosis and Treatment of Primary Liver Cancer in China (2017 Edition). Liver Cancer. 2018; 7(3): 235-60.

5. Cheng S, Chen M, Cai J,et al. Chinese Expert Consensus on Multidisciplinary Diagnosis and Treatment of Hepatocellular Carcinoma with Portal Vein Tumor Thrombus (2018 Edition). Liver Cancer. 2020; 9(1): 28-40.

6. Lee IJ, Lee JH, Lee YB, et al. Effectiveness of drug-eluting bead transarterial chemoembolization versus conventional transarterial chemoembolization for small hepatocellular carcinoma in Child-Pugh class A patients. Ther Adv Med Oncol. 2019; 11:432486184.

7. Zhang S, Huang C, Li Z, et al. Comparison of pharmacokinetics and drug release in tissues after transarterial chemoembolization with doxorubicin using diverse lipiodol emulsions and CalliSpheres Beads in rabbit livers. Drug Deliv. 2017; 24(1): 1011-17.

8. Wang H, Wang J, Lv T, et al. Loadability and Releasing Profiles In Vitro and Pharmacokinetics In Vivo of Vinorelbine and Raltitrexed by CalliSpheres Beads. Cancer Biother Radiopharm. 2020.

9. Han X, Chen Q, Sun Y, et al. Morphology, Loadability, and Releasing Profiles of CalliSpheres Microspheres in Delivering Oxaliplatin: An In Vitro Study. Technol Cancer Res Treat. 2019; 18:1078145637.

10. Zhou GH, Han J, Sun JH, et al. Efficacy and safety profile of drug-eluting beads transarterial chemoembolization by CalliSpheres(R) beads in Chinese hepatocellular carcinoma patients. Bmc Cancer. 2018; 18(1): 644.

11. Wang CY, Xia JG, Yang ZQ, et al. Transarterial chemoembolization with medium-sized doxorubicin-eluting Callisphere is safe and effective for patients with hepatocellular carcinoma. Sci Rep. 2020; 10(1): 4434

12. Bruix J, Sherman M. Management of hepatocellular carcinoma. Hepatology. 2005; 42(5): 1208-36.

13. Yoon SM, Lim YS, Won HJ, et al. Radiotherapy plus transarterial chemoembolization for hepatocellular carcinoma invading the portal vein: long-term patient outcomes. Int J Radiat Oncol Biol Phys. 2012; 82(5): 2004-11.

14. Moriguchi M, Furuta M, Itoh Y. A Review of Non-operative Treatments for Hepatocellular Carcinoma with Advanced Portal Vein Tumor Thrombus. J Clin Transl Hepatol. 2017; 5(2): 177-83.
15. Zhang ZM, Lai EC, Zhang C, et al. The strategies for treating primary hepatocellular carcinoma with portal vein tumor thrombus. Int J Surg. 2015; 20:8-16.

16. Luo J, Guo RP, Lai EC, et al. Transarterial chemoembolization for unresectable hepatocellular carcinoma with portal vein tumor thrombosis: a prospective comparative study. Ann Surg Oncol. 2011; 18(2): 413-20.

17. Lv WF, Liu KC, Lu D, et al. Transarterial chemoembolization for hepatocellular carcinoma combined with portal vein tumor thrombosis. Cancer Manag Res. 2018; 10:4719-26.

18. Bruix J, Raoul JL, Sherman M, et al. Efficacy and safety of sorafenib in patients with advanced hepatocellular carcinoma: subanalyses of a phase III trial. J Hepatol. 2012; 57(4): 821-29.

19. Zhong JH, Rodriguez AC, Ke Y, et al. Hepatic resection as a safe and effective treatment for hepatocellular carcinoma involving a single large tumor, multiple tumors, or macrovascular invasion. Medicine (Baltimore). 2015; 94(3): e396.

20. Zhang XP, Wang $\mathrm{K}, \mathrm{Li} \mathrm{N}$, et al. Survival benefit of hepatic resection versus transarterial chemoembolization for hepatocellular carcinoma with portal vein tumor thrombus: a systematic review and meta-analysis. Bmc Cancer. 2017; 17(1): 902.

21. Facciorusso A, Di Maso M, Muscatiello N. Drug-eluting beads versus conventional chemoembolization for the treatment of unresectable hepatocellular carcinoma: A meta-analysis. Dig Liver Dis. 2016; 48(6): 571-77.

22. Aliberti $C$, Carandina $R$, Lonardi $S$, et al. Transarterial Chemoembolization with Small Drug-Eluting Beads in Patients with Hepatocellular Carcinoma: Experience from a Cohort of 421 Patients at an Italian Center. J Vasc Interv Radiol. 2017; 28(11): 1495-502.

23. Chen $\mathrm{P}$, Yuan $\mathrm{P}$, Chen $\mathrm{B}$, et al. Evaluation of drug-eluting beads versus conventional transcatheter arterial chemoembolization in patients with unresectable hepatocellular carcinoma: A systematic review and meta-analysis. Clin Res Hepatol Gastroenterol. 2017; 41(1): 75-85.

24. Huang $K$, Zhou Q, Wang $R$, et al. Doxorubicin-eluting beads versus conventional transarterial chemoembolization for the treatment of hepatocellular carcinoma. J Gastroenterol Hepatol. 2014; 29(5): 920-25.

25. Liu YS, Ou MC, Tsai YS, et al. Transarterial chemoembolization using gelatin sponges or microspheres plus lipiodol-doxorubicin versus doxorubicin-loaded beads for the treatment of hepatocellular carcinoma. Korean J Radiol. 2015; 16(1): 125-32

26. Akinwande O, Kim D, Edwards J, et al. Is radioembolization ((90)Y) better than doxorubicin drug eluting beads (DEBDOX) for hepatocellular carcinoma with portal vein thrombosis? A retrospective analysis. Surg Oncol. 2015; 24(3): 270-75.

27. Gorodetski B, Chapiro J, Schernthaner R, et al. Advanced-stage hepatocellular carcinoma with portal vein thrombosis: conventional versus drug-eluting beads transcatheter arterial chemoembolization. Eur Radiol. 2017; 27(2): 526-35.

28. Wu B, Zhou J, Ling G, et al. CalliSpheres drug-eluting beads versus lipiodol transarterial chemoembolization in the treatment of hepatocellular carcinoma: a short-term efficacy and safety study. World J Surg Oncol. 2018; 16(1): 69.

29. Sun JH, Zhou T, Zhu T, et al. Portal Vein Stenting Combined with Iodine-125 Seeds Endovascular Implantation Followed by Transcatheter Arterial Chemoembolization for Treatment of Hepatocellular Carcinoma Patients with Portal Vein Tumor Thrombus. Biomed Res Int. 2016; 2016(3048261). 